\title{
Maternal diet during pregnancy and child weight outcomes
}

\author{
Leonie Helen Bogl ${ }^{1,2}$, Susannne Strohmaier ${ }^{1}$, Heather Eliassen ${ }^{3,4}$, Jennifer Massa ${ }^{5}$, Alison Field ${ }^{6}$, \\ Jorge Chavarro $^{3,7}$, Ming Ding ${ }^{4}$, Rulla Tamimi ${ }^{3,4}$ and Eva Schernhammer ${ }^{1}$ \\ ${ }^{1}$ Department of Epidemiology, Center for Public Health, Medical University of Vienna, Vienna, Austria, \\ ${ }^{2}$ Leibniz Institute for Prevention Research and Epidemiology - BIPS, Bremen, Germany, \\ ${ }^{3}$ Channing Division of Network Medicine, Brigham and Women's Hospital and Harvard Medical School, Boston, USA, \\ ${ }^{4}$ Department of Epidemiology, Harvard T.H. Chan School of Public Health, Boston, USA, \\ ${ }^{5}$ Department of Nutrition, Harvard T.H. Chan School of Public Health, Boston, USA, \\ ${ }^{6}$ Department of Epidemiology, Brown University, Providence, RI, USA and \\ ${ }^{7}$ Department of Epidemiology, Harvard T.H. Chan School of Public Health, Boston, USA
}

\begin{abstract}
Introduction: Animal studies suggest that maternal diet during pregnancy influences susceptibility to obesity in the next generation. One-carbon nutrients involved in epigenetic processes represent a possible underlying mechanism. Unfortunately, there is limited research in humans to support animal findings, and the few studies available have been limited to birth defects and weight outcomes in early infancy. The aim of this project was to examine the associations between maternal diet (diet quality indicators and one-carbon nutrients) during the period surrounding pregnancy and long-term weight outcomes in the offspring.
\end{abstract}

Methods: We examined 2,729 mother-child pairs from the Nurses' Health Study II and the offspring cohort Growing Up Today Study 2 (GUTS 2). Children, 12-14 years at baseline were 21-23 years at the last follow-up. Overweight was defined for youth less than 18 years of age using sex- and age-specific body mass index (BMI) cut-offs recommended by the International Obesity Task Force and for those 18 years or older as BMI $\geq 25 \mathrm{~kg} / \mathrm{m}^{2}$. Dietary information was collected by a validated 131-item food frequency questionnaire (FFQ) and questions on supplement use. Maternal dietary patterns were calculated from FFQs using three diet quality scores the alternate Healthy Eating Index (aHEI), Alternate Mediterranean Diet (aMED) and Dietary Approach to Stop Hypertension (DASH). Log-binomial models were used to estimate relative risks (RRs) and 95\% confidence intervals.

Results: In models adjusted for sex, gestational age at delivery and maternal total energy intake, greater maternal adherence to aMED and DASH, but not aHEI, was associated with lower overweight risk in the offspring $\left(\mathrm{RR}_{\mathrm{Q} 5 \text { vs } \mathrm{Q} 1}=0.82[0.70-0.97]\right.$ for aMED and 0.86 [0.72-1.04] for DASH) (P for trend $<0.05$ for both). After additional adjustment for maternal pre-pregnancy BMI and sociodemographic characteristic, none of the diet quality scores was significantly associated with child's birth weight or overweight risk during follow-up. Analysis with one-carbon nutrients are ongoing; we hypothesize that children born to mothers with greater intake of one-carbon nutrients have a lower risk of developing overweight compared to children of mothers with lower intake of these nutrients.

Conclusions: Maternal diet quality indices during pregnancy were not associated with child's birth weight or the risk of developing overweight at ages 12 to 23 years. Further research on maternal dietary intake during pregnancy is warranted because pregnancy offers a window of opportunity to promote behavioral change that may program the health of the next generation.

\section{Conflict of Interest}

There is no conflict of interest 